\title{
Motor responses of the small intenstine to intraluminal distension in normal volunteers and a patient with visceral neuropathy
}

\author{
G P N KENDALL, D G THOMPSON, AND S J DAY \\ From the Department of Medical Research, St Mark's Hospital, London, and Department of Gastroenterology \\ and Biometry, The London Hospital Medical College, London.
}

SUMmARY The motor responses of the small intestine to intraluminal distension were studied proximal and distal to an inflatable balloon in 13 normal volunteers. During fasting, distension rapidly induced a persistent localised inhibition of distal contractile activity with a small proximal increase. Proximally, phase III activity was unaffected during distension but its propagation across and appearance below the balloon was inhibited. Upon deflating the balloon a normal motor pattern rapidly returned. Similar changes were observed during distension in the fed state. The changes in the motor pattern resemble those of the intrinsically mediated 'peristaltic reflex', studied in animals, and suggest that in man the response to balloon distension may also be mediated through an intrinsic mechanism. A patient with a visceral neuropathy, studied in a similar manner, had no inhibition of distal motor activity during distension, suggesting a functional defect of the enteric nerves. Further observations of the motor responses to distension in similar patients seem indicated to determine the usefulness of this technique for evaluating enteric nervous system function when an abnormality is suspected.

In their classic paper, of 1899, ' Bayliss and Starling described an alteration of intestinal motor activity after the introduction of a distending bolus into the extrinsically denervated jejunum of anaesthetised dogs. They observed a proximal increase, and distal reduction, in contractile activity as the bolus progressed along the bowel, a pattern they considered to represent the mechanism of peristalsis. The nature of their experimental preparation, together with the rapidity of onset and localisation of the response led them to conclude that these motor changes were mediated via the intrinsic enteric nerves. This response, which they termed the law of the intestine', has subsequently become known as the 'peristaltic reflex': ' More recently, studies in isolated intestine have confirmed mediation of the response through the enteric nervous system "' and delineated the neural pathways involved; distension of the

Address for correspondence: Dr D G Thompson. Department of Medicine. Hope Hospital. Eccles Old Road. Salford M68 HID.

Received for publication 3 October. 1986. intestine induces ascending excitatory and descending inhibitory discharges. Studies of the innervated canine jejunum have also produced similar changes showing that the response also occurs in intact animals. ${ }^{+}$

Abnormalities of the enteric nervous system are now being recognised in patients. Such patients present with abdominal pain, distension and vomiting and form part of the syndrome of chronic idiopathic intestinal pseudo-obstruction. At present the diagnosis of visceral neuropathy is clinically based and confirmation requires a laparotomy with full thickness biopsy of the small intestine, a procedure which is often clinically inappropriate. Investigation of suspected enteric nervous system dysfunction by manometric studies of the small intestine has been attempted" but the results, even in histologically proven cases, are usually insufficiently discriminative to be diagnostic.' Because the peristaltic reflex is known to be mediated via the enteric nervous system we decided to undertake a series of investigations to characterise the responses of the 
intact human small intestine to local distension. We also studied the motor responses of a patient with a visceral neuropathy in order to assess the possible use of intraluminal distension as a diagnostic aid in such patients.

\section{Methods}

SUBJECTS

The protocols for the studies undertaken were approved by the London Hospital Ethics Committee in February 1984. The normal subjects were healthy adult volunteers (age 19-28 years), without history of gastrointestinal disorder, who gave their informed consent before the study. A 35 year old female patient with a clinical diagnosis of neuropathic intestinal pseudo-obstruction was also studied in a similar manner.

Small intestinal intraluminal pressure activity was recorded using a standard multilumen perfused tube technique. ${ }^{\times}$The tube was constructed from eight radiolucent polyvinyl chloride (PVC) capillary tubes (internal diameter (ID) $0.63 \mathrm{~mm}$, external diameter (ED) $1.4 \mathrm{~mm}$ ), bonded, with tetrahydrofuran, around a central radio-opaque PVC tube (ID $2 \mathrm{~mm}$, ED $2.5 \mathrm{~mm}$ ). A balloon, made from a $5 \mathrm{~cm}$ length of condom rubber, was attached $25 \mathrm{~cm}$ proximal to the distal end of the tube and inflated with an additional PVC tube (ID $1 \mathrm{~mm}$, ED $1.6 \mathrm{~mm}$ ) (Fig 1).

After an overnight fast, the tube was swallowed and allowed to pass under fluoroscopic control into the upper jejunum until the balloon lay distal to the ligament of Treitz. Each channel was then perfused with distilled water at $0.3 \mathrm{ml}$ per minute using a low compliance pneumohydraulic perfusion pump. Intraluminal pressure changes were measured at multiple sites, proximal and distal to the balloon, using strain gauge transducers (Gaeltec S8b, Skye, Scotland) attached to the proximal end of each lumen. The pressure changes were recorded on an eight channel chart recorder (Watanabe Linear Corder mark VII, Tokyo, Japan). Intestinal distension was achieved by inflating the balloon until abdominal discomfort was just felt. Persistence of inflation throughout the test period was confirmed by comparing the volume of air removed at the end of the test period with that instilled at the beginning.

EXPERIMENTAI, PROTOCOL.

Fasted studies

Eight subjects underwent two consecutive, randomly ordered, three hour periods of study to examine the effects of distension on fasted motor patterns. During the control period the balloon was deflated but for the test period it was inflated, as described above.

In three subjects additional studies were done in

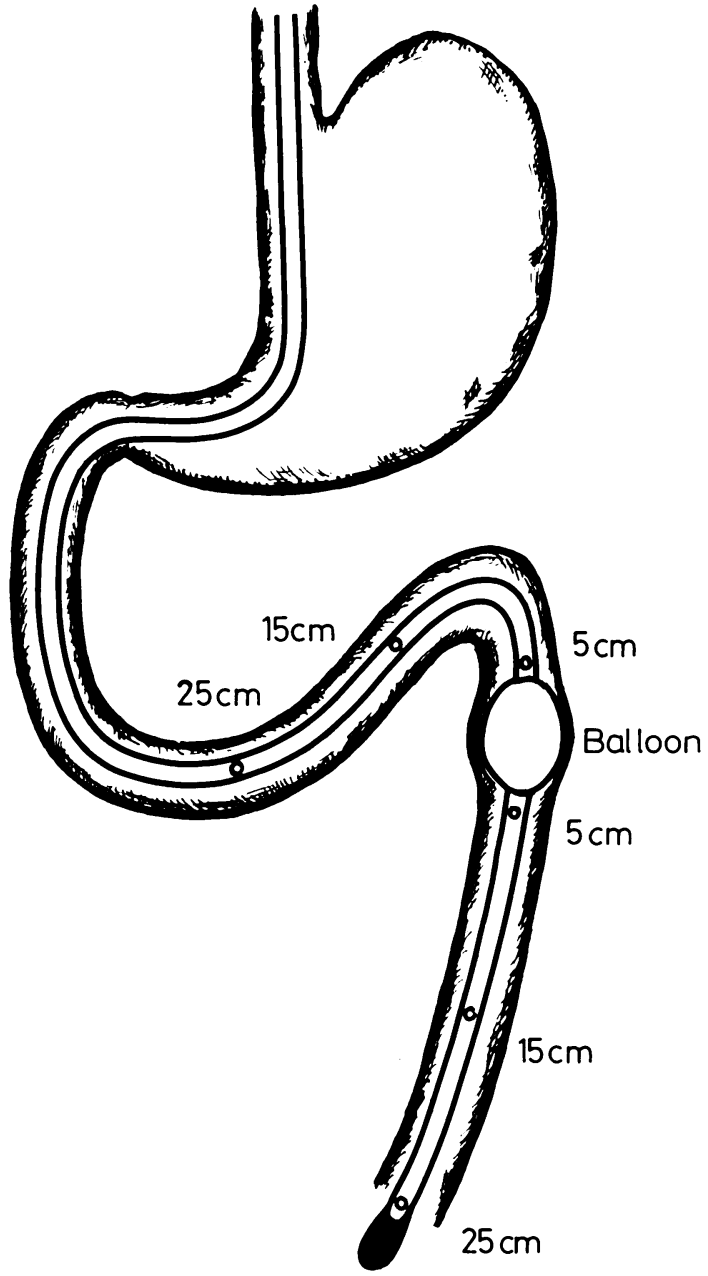

Fig. 1 Diagram of the multilumen perfused tube positioned in the upper jejunum so that the balloon is distal to the ligament of Treitz. Positions of the perfusion ports and their relationship to the balloon are indicated.

which the balloon was inflated for short periods while phase III activity was present in the test segment.

\section{Fed studies}

The effect of intraluminal distension on fed activity was studied in the five subjects after a liquid mixed nutrient meal (cream of chicken soup; HJ Heinz Ltd, $400 \mathrm{ml}, 225 \mathrm{cal}$ ), ingested during the phase II of fasted activity. Twenty minutes after meal ingestion the balloon was inflated for 30 minutes. Recording continued for a further 30 minutes after deflation.

DATA ANALYSIS

The small intestinal intraluminal pressure recordings were examined by direct inspection. In the fasted 
studies, the different phases of activity were identified" and the migration of phase III was noted. The number of contractions per minute were then counted in all channels for the separate study periods. In the fed studies the number of contractions per minute were counted for the periods before, during, and after distension. To minimise inclusion of artefact only pressure changes greater than $5 \mathrm{~mm}$ of mercury were included and any activity that occurred simultaneously in all channels, likely to have been caused by raised intra-abdominal pressure or tube movement, was excluded. The differences between the number of contractions above and below the balloon with and without distension were calculated for each study period. In the fed studies a comparison of activity before and after distension was also done. Analysis of variance" was used to determine the probability that the observed changes could have occurred by chance.

\section{Results}

NORMAL SUBJECTS

Fasted studies

In the control period all subjects showed the normal triphasic motor pattern with phase III starting in the proximal duodenum and migrating aborally. ${ }^{9}$ During the test period balloon inflation caused transient abdominal discomfort in all subjects, which disappeared within 5-10 minutes, (volume of inflation: median $33 \mathrm{ml}$, range $28-52 \mathrm{ml}$, balloon diameter $2 \cdot 7-3.6 \mathrm{~cm})$. Balloon distension started during phase I in three subjects and phase II in five. When motor activity was present, inflation was followed by a rapid and marked reduction in contractile activity distal to the balloon $(p<0.05)$ (Fig. 2). This was most prominent in the channel closest to the balloon and persisted for the duration of the distension (Fig. 3).

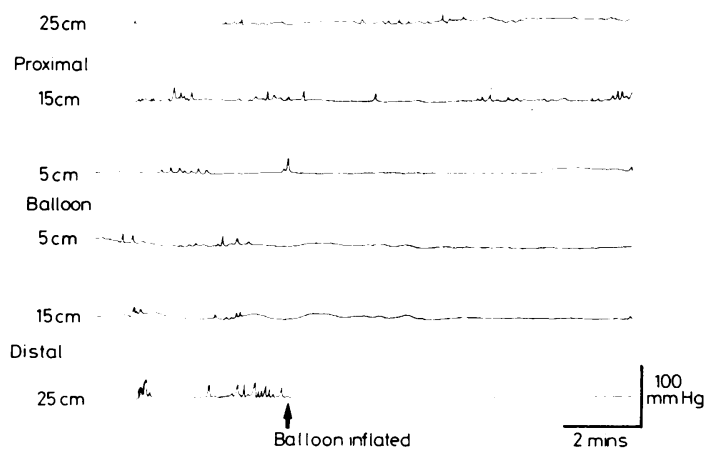

Fig. 2 Diagram shows a tracing of the typical changes in fasted small intestinal intraluminal pressure activity associated with balloon distension in a normal subject. Inhibition of distal contractile activity is seen shortly after inflation.
The caudal extent of the inhibition varied between individuals but was never less than $15 \mathrm{~cm}$.

Proximal to the distension small intestinal motility altered, with an increase in the proportion of phase II activity from $58 \pm 4 \%$ (mean \pm SEM) during the control period to $82 \pm 6 \%$ during distension. There was also an increase in the mean number of contractions during phase II changing from $14 \cdot 2 \pm 2$ per 10 minutes during the control period to $24 \cdot 6 \pm 3 \cdot 1$ during distension. Because of the relatively small number of subjects studied, however, and because of the known marked intersubject variation in fasted motor activity" the overall changes in proximal motor activity did not reach statistical significance by the analytical method used. Reference to Figure 3, however, will show the consistent upward trend in motor activity in all proximal channels.

During distension phase III activity occurred proximal to the balloon at a frequency equal to that in the undistended state. The incidence of proximal phase III activity per three hours expressed as the median and (range) was $2(1-3)$ for the control period and $2(1-3)$ for the test period. Phase III activity was never seen to traverse, or appear below, the inflated balloon. On the three additional occasions when distension was induced during phase III it continued above but was inhibited below the point of distension.

Upon balloon deflation proximal and distal motor activity rapidly returned to normal in all but one subject in whom the inhibition persisted for at least 30 minutes after deflation.

\section{Fed studies}

After meal ingestion the fasted pattern was replaced by irregular contractile activity in all subjects. As in the fasted state, distension caused a rapid and persistent reduction in distal contractile activity over a distance of at least $25 \mathrm{~cm}(p<0.02)$, the effect being greatest in the channel nearest the balloon (Fig. 4). No change in proximal contractile activity was seen. After balloon deflation the distal motor inhibition promptly returned to normal. There was no significant difference in contractile frequency between the predistension and postdistension periods.

\section{CASE REPORT}

A 35 year old female patient presented with a two year history of intermittent colicky abdominal pain associated with a feeling of abdominal distension and palpable intestinal contraction, usually precipitated by the ingestion of food. She also complained of difficulty with and infrequency of defecation, opening her bowels every three to six days with the aid of laxatives, together with difficulty in swallowing food and reflux of gastric contents. Achalasia of the oesophagus had been diagnosed manometrically 17 


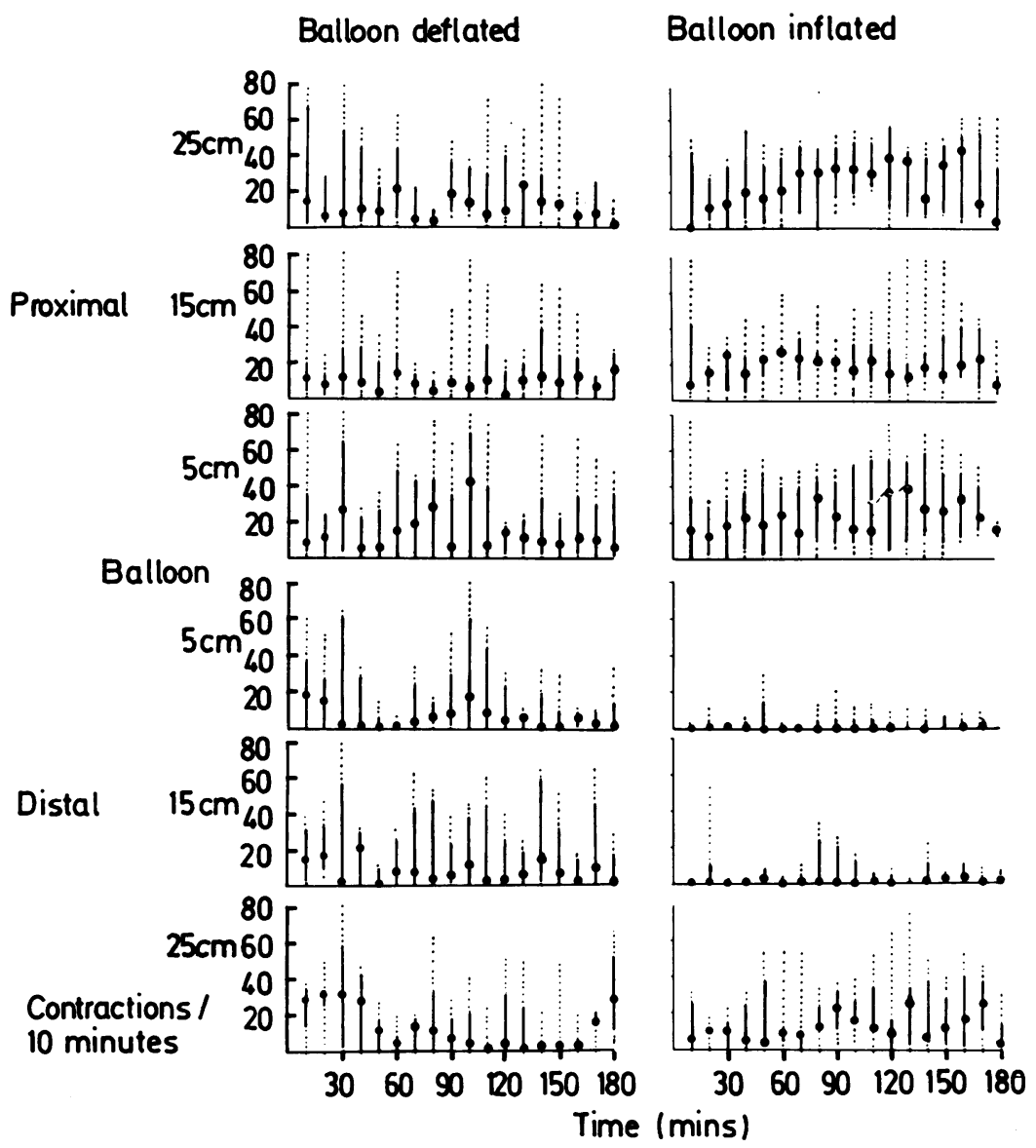

Fig. 3 Graphs show the frequency of contractions/10 minutes in the fasted state for all the normal subjects. Channels 5, 15 and $25 \mathrm{~cm}$ either side of the balloon are shown during the control period (balloon deflated) and the test period (balloon inflated). Plots indicate the median $\bullet$, interquartile range —_ and range ---.--.. During inflation there was a significant and marked reduction in the number of contractions in the channels 5 and $15 \mathrm{~cm}$ below the balloon, $p<0.05$ (analysis of variance). An overall increase in proximal activity was also seen, but this did not reach statistical significance.

years previously and had been treated by two Heller's operations. There was no history of cardiovascular or neurological disturbance, no history of previous medical disorders, no known family history of gastrointestinal problems, and apart from laxatives she took no medication.

On examination she was in good general health. No abnormal neurological or cardiovascular signs were found, and in particular there was no postural hypotension or alteration in vasomotor tone or sweating to indicate an autonomic neuropathy. Examination of the abdomen revealed no abnormality except that deep palpation on several occasions induced palpable and persistent contraction of the intestine beneath the hand.
Laboratory investigations revealed a normal full blood count and biochemical profile. Plain abdominal radiographs never revealed an increased intestinal gas and no evidence of oesophageal, gastric or intestinal dilatation was demonstrable by contrast radiography. A full thickness biopsy of the rectum showed an absence of ganglion cells, indicating a diagnosis of intestinal neuropathy, but histochemical staining, with acetylcholinesterase, and the presence of a rectosphincteric distension reflex excluded Hirschprung's disease.

\section{Small intestinal manometry}

The small intestinal motor activity of the patient was studied in a similar manner to the normal subjects, 
using a tube modified by the addition of perfusion channels 40 and $50 \mathrm{~cm}$ distal to the balloon. During a three hour period of fasted recording, four episodes of phase III activity were seen. On one occasion

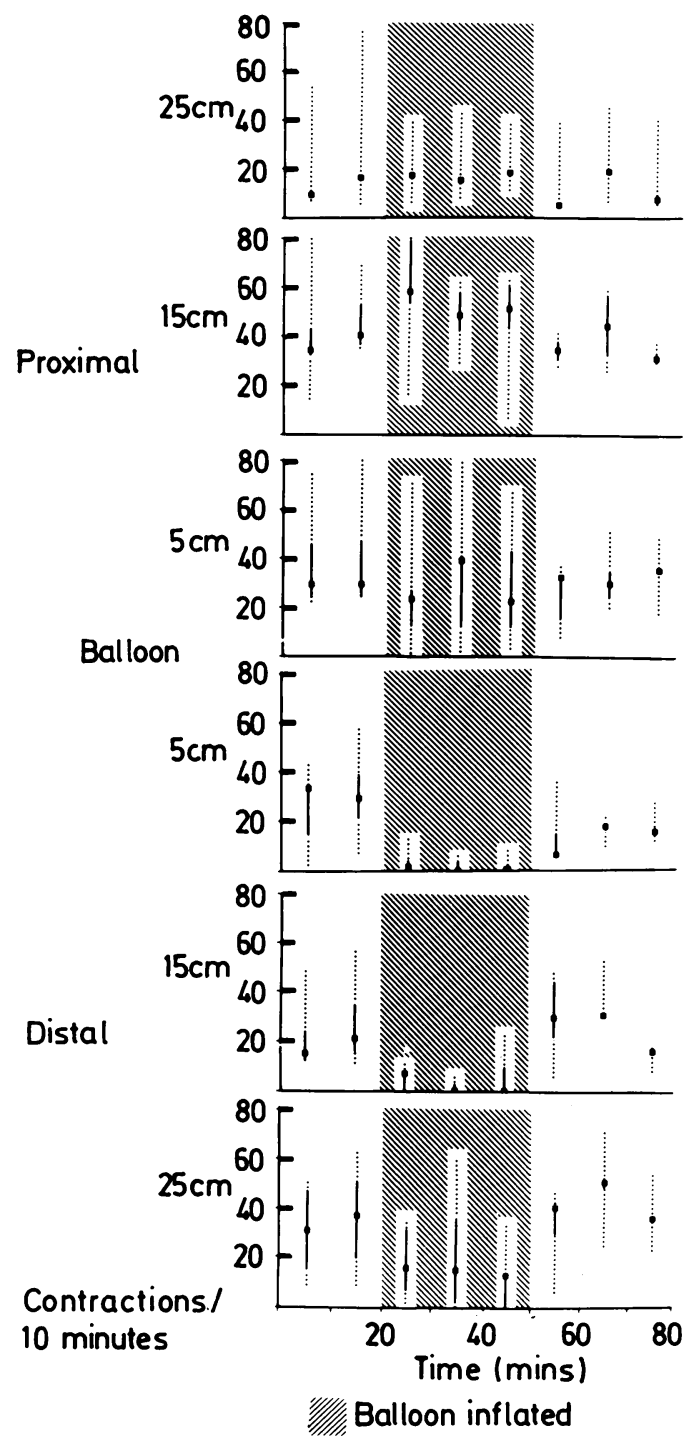

Fig. 4 Graphs shows the number of contractions/10 minutes during the fed state for all the subjects. The channels 5,15 and $25 \mathrm{~cm}$ either side of the balloon are shown during the control periods (balloon deflated) and the test period (balloon inflated). Plots show the median , interquartile range - and range -...-.-. Upon balloon inflation, 20 minutes after meal ingestion, there was a marked reduction in the number of contractions below the balloon, $p<0.02$ (analysis of variance). This change was most noticeable in the channels closest to the balloon. No alteration in motor activity was seen above the balloon. phase III started proximally and migrated aborally, in sequence, through all the channels. On the other occasions, however, phase III activity was abnormal, migrating only over short distances.

Inflation of the balloon in the upper jejunum induced transient discomfort as in the normal subjects (volume of balloon inflation $60 \mathrm{ml}$, diameter $3.9 \mathrm{~mm}$ ). In contrast with the normal subjects, no inhibition of motor activity below the balloon was seen, but a rapid and marked increase in the number of contractions occurred both above and below the point of distension. These changes were associated with the development of abdominal pain and the occurrence of visible and palpable intestinal contractions, similar to those experienced on other occasions. Three periods of balloon distension, lasting between six and nine minutes, were performed at 10-20 minute intervals and each time the same response was observed. Initial inspection of the tracing suggested that the pressure changes might have been simultaneous and therefore caused by a common cavity phenomenon. The possibility of extraintestinal artefact, such as body movement or tube displacement, was considered but all attempts to exclude these factors were unsuccessful in altering the motor pattern. Closer inspection of the tracing revealed contractions below the distended balloon which were not visible above indicating failure of distal inhibition. Furthermore during these episodes an intravenous injection of hyoscine butylbromide $(20 \mathrm{mg})$ was given and this abolished the increased activity indicating that the observed response was indeed intestinal in origin. Ingestion of a soup meal produced her typical symptoms together with palpable intestinal contractions. Balloon inflation during the fed state failed to induce the distal motor inhibition seen in the normal subjects (Fig. 5). Upon balloon deflation the motor activity returned to the preinflation pattern over a period of five to 10 minutes and the symptoms slowly subsided.

\section{Discussion}

Our results show that intraluminal distension in the normal intact human small intestine induces a marked reduction in distal motor activity with an increase in proximal activity. Although our experimental protocol, which used prolonged distension, differs from that previously used to produce the peristaltic reflex,,$^{1-3}$ the similarity of the motor pattern suggests the changes may be mediated through similar intrinsic pathways.

During the investigation of experimental small intestinal obstruction Summers et al' found similar changes to those seen by us, with a proximal increase and distal inhibition in myoelectric activity when a 


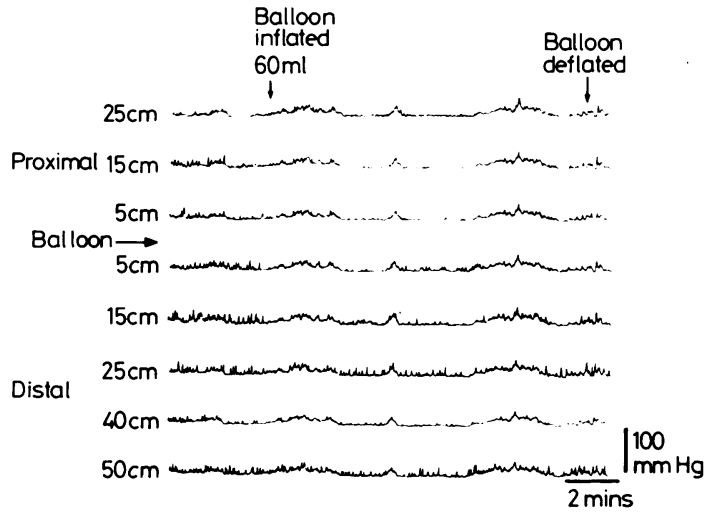

Fig. 5 Diagram shows a tracing of fed intestinal motor activity from the patient to show the effect of balloon distension. In contrast with the pattern seen in normal subjects, motor activity continues below the site of distension.

balloon was inflated to obstruct intraluminal flow of nutrients. Prihoda et al ${ }^{4}$ studied the nature of this response to obstruction in greater detail. They noted that the reduction in myoelectric activity below an occlusive intraluminal balloon was initially localised immediately below the balloon but spread progressively along the distal small intestine as the study continued. This spread of motor activity was not seen in our fed studies but our period of postprandial distension was only of 30 minutes duration and an increase in distal inhibition might have been seen if the distension had been more prolonged.

In vitro evidence implicates intestinal stretch, ${ }^{13}$ detected by intramural tension receptors, ${ }^{14}$ as the trigger for the response to distension. Pharmacological studies ${ }^{2+}$ indicate that the proximal excitation is cholinergically mediated while the descending inhibition occurs through a non-adrenergic, noncholinergic pathway, vasoactive intestinal polypeptide being a likely candidate for the transmitter. ${ }^{15}$

Although the motor changes which we observed closely resemble those observed during in vitro studies, other possibilities must be considered. It could be hypothesised, for example, that during feeding a lack of intraluminal contents below the balloon might cause the reduced activity. The rapidity of the inhibitory response, however, which began within seconds of distension, must make this unlikely. Prihoda et $\mathrm{al}^{4}$ also showed that a lack of intestinal contents was unlikely to be responsible for the distal inhibition as it persisted when luminal contents were reinfused below the balloon. It could also be hypothesised that pain induced by distension could have caused the inhibition. Certainly severe somatic pain does inhibit small intestinal activity but the effects are generalised. ${ }^{16}$ Furthermore in our subjects the discomfort was mild and transient and for most of the studies subjects experienced no abdominal discomfort at all.

The inhibition of phase III migration during distension provides further evidence for the control of its propagation by an intrinsic pathway. Although an initial report suggested that the migrating motor complex was propagated via the extrinsic nerves, ${ }^{17}$ subsequent studies using intestinal transection and reanastomosis, ${ }^{18}$ autotransplantation, ${ }^{19}$ and local intra-arterial injection of nerve blocking drugs ${ }^{211}$ have shown the overwhelming importance of the intrinsic enteric nerves. Furthermore migratory phase III-like activity has been demonstrated in autotransplanted human jejunum indicating that the mechanism in man is also intrinsic. ${ }^{21}$

The results of intraluminal distension in our patient contrast markedly with those of our normal subjects. The apparent failure of motor inhibition below the distending balloon suggests that, in this patient, the descending inhibitory pathway of the myenteric plexus is defective. It is possible to speculate that this could be the result of variation in the degree of distension but this seems unlikely as the balloon was distended to a greater volume than in the normal subjects and the sensory end point was similar. Furthermore other studies done in our laboratory (unpublished data) indicate that the pattern of response seen is not dependent upon degrees of distension but remains consistent so long as a certain threshold, which is less than that required for perception of the distension, is exceeded. The increase in apparently simultaneous contractile activity seen during distension may represent contractions transmitted through a closed loop system with spasm of the intestine at either end of the manometry tube. Whatever the reason such activity was never seen in any of our other studies and was rapidly abolished by cholinergic blockade indicating an abnormal motor response to distension. Further studies in patients with histologically proven visceral neuropathy, however, are required to confirm these findings.

Although it is tempting to speculate about the relationship between this abnormality and the nature of her symptoms, our data do not allow any firm conclusions to be made and other functional disturbances of the gut could be contributary. Our results, however, do suggest that the demonstration of an abnormal response to intraluminal distension might provide a convenient guide to enteric nerve disease, just as demonstration of abnormal somatic reflexes indicates somatic nerve damage. If confirmed in other patients, an abnormal distension reflex may prove diagnostically superior to previous manometric methods. These have largely relied on the recognition of phase III abnormalities, "which as was demonstrated in our patient are often intermittent. A 
further potential advantage of distension is convenience; an abnormality would be rapidly recognised while identification of phase III abnormality may require prolonged intubation.

In conclusion, our experiments have shown that the normal human small intestine responds in a characteristic way to intraluminal distension. This response resembles that seen in the isolated intestine, indicating that it is also likely to be mediated via an intrinsic neural pathway. If confirmed, the demonstration of abnormalities of this pattern in patients with intestinal pseudo-obstruction would allow a more definite clinical diagnosis of impaired myenteric plexus function than has previously been possible.

Dr Kendall is the WEG Knott Research Fellow of the British Digestive Foundation. Dr Thompson is a Wellcome Trust Senior Lecturer in Medicine. We would like to thank Dr G Sladen of Guy's Hospital, London, for allowing us to study and report his patient, Professor Lennard-Jones for his help in preparing this manuscript and Miss E Walker for her help in performing the studies.

\section{References}

1 Bayliss WM, Starling EH. The movements and innervation of the small intestine. J Physiol 1899; 24: 99-143.

2 Kottegoda SR. An analysis of possible nervous mechanisms involved in the peristaltic reflex. J Physiol 1969; 200: 687-712.

3 Holman ME. The intrinsic innervation and peristaltic reflex of the small intestine. In: Bulbring $E$, Brading AF, Jones AW, Tomita T, eds. Smooth muscle: assessment of current knowledge. 1st ed. London: Edward Arnold Press, 1981: 311-38.

4 Prihoda M, Flatt A, Summers RW. Mechanism of motility changes during acute intestinal distension in the dog. Am J Physiol 1984; 247: G37-G42.

5. Schuffler MD. Chronic intestinal pseudo-obstruction syndromes. Med Clin North Am 1981; 65: 1331-58.

6 Stanghellini V, Camilleri M, Magagelada J-R. Chronic idiopathic intestinal pseudo-obstruction, diagnostic importance of gastrointestinal motility. [Abstract] Gastroenterology 1985; 88: A 1596.

7 Malagelada JR, Stanghellini V. Manometric evaluation of functional upper gut symptoms. Gastroenterology 1985; 85: 1223-31.

8 Arndorfer RC, Stef JJ, Dodds WJ, Linehan JH, Hogan WJ. Improved infusion system for intraluminal esophageal manometry. Gastroenterology 1977; 73: 23-7.

9 Code CF, Marlett JA. The interdigestive myo-electric complex of the stomach and small intestine of dogs. J Physiol 1975; 246: 289-309.

10 Armitage P. Statistical methods in medical research. 1st ed. Oxford: Blackwell Scientific Publications, 1971.

11 Kerlin P, Phillips S. Variability of motility of the ileum and jejunum in healthy humans. Gastroenterology 1982; 82: $694-700$.

12 Summers RW, Yanda R, Prihoda M, Flatt A. Acute intestinal obstruction: An electromyographic study in dogs. Gastroenterology 1983; 85: 1301-6.

$13 \mathrm{Ginzel} \mathrm{KH}$. Investigation concerning the initiation of the peristaltic reflex in the guinea-pig ileum. J Physiol 1959; 148: P75-P76.

14 Iggo $\mathrm{A}$. Tension receptors in the stomach and the urinary bladder. J Physiol 1955; 128: 593-607.

15 Grider JR, Makhlouf GM. VIP antiserum inhibits caudal relaxation and augments orad contraction of intestinal peristaltic reflex. [Abstract] Gastroenterology 1985; 88: A1406.

16 Stanghellini V, Magagelada J-R, Zinsmeister AR, Go VLW, Kao PC. Stress induced gastroduodenal motor disturbance in humans: possible humoral mechanisms. Gastroenterology 1983; 85: 83-91.

17 Carlson GM, Bedi BS, Code CF. Mechanism of propagation of intestinal interdigestive myoelectric complex. Am J Physiol 1972; 222: 1027-30.

18 Sarna S, Condon RE, Cowles V. Enteric mechanisms of initiation of migrating myoelectric complexes in dogs. Gastroenterology 1983; 84: 814-22.

19 Sarr MG, Kelly KA. Myoelectrical activity of the autotransplanted canine jejunoileum. Gastroenterology 1981; 81: 303-10.

20 Sarna S, Stoddard C, Belbeck L, McWade D. Intrinsic nervous control of migrating myoelectric complexes. Am J Physiol 1981; 241: G16-G23.

21 Kerlin P, Harris B. The function of a free jejunal graft in the cervical oesophagus. [Abstract] Gastroenterology 1985; 88: A 1443. 